Einführung zum Thema

Onkologe 2020 $26: 2-3$

https://doi.org/10.1007/s00761-019-00700-w

(c) Springer Medizin Verlag $\mathrm{GmbH}$, ein Teil von Springer Nature 2020

\section{Heinz-Peter Schlemmer' · P. M. Schlag ${ }^{2}$}

${ }^{1}$ Abt. Radiologie, Deutsches Krebsforschungszentrum, Stiftung des öffentlichen Rechts, Heidelberg, Deutschland

${ }^{2}$ Charité Comprehensive Cancer Center, Berlin, Deutschland

\title{
Erweiterte digitale Bildgebung und molekulare Diagnostik - Aufbruch zu neuen Ufern in der Onkologie
}

Bildgebung ist von essenzieller Bedeutung für die Onkologie. Das möglichst frühzeitige Erkennen eines Tumors, dessen genaue anatomische Zuordnung in Relation zu den umgebenden gesunden Geweben, die Darstellung der lokalen Tumorausbreitung mit möglicher Umgebungsinfiltration, die Visualisierung des biologischen und heterogenen Wachstumsverhaltens sowie das frühe Erkennen möglicher distanter Streuherde sind wichtig für die Planung einer individuellen Behandlung und die damit verbundene spätere Prognose. Bereits die pathologische Diagnose einer Krebserkrankung basiert häufig auf der Präzision einer Gewebeprobe, welche durch eine bildgebungsgesteuerte Biopsie gewonnen wurde. Ebenso werden für die Durchführung der jeweiligen Therapie, für das Therapiemonitoring und für Verlaufskontrollen zur frühzeitigen Erkennung von Rezidiven oder Metastasen bildgebende Verfahren benötigt. Doch nicht nur die Diagnostik der Krebserkrankung selbst, sondern auch die Diagnostik aller anderen, tumorbegleitenden sowie therapiebezogenen Erkrankungen erfordert häufig ein bildgebendes Verfahren.

Auf Grundlage aller vorliegenden klinischen und laborchemischen Befunde sowie insbesondere der Ergebnisse bildgebender und histologischer Untersuchungen wird das weitere therapeutische Vorgehen individuell in einer interdisziplinären Tumorkonferenz geplant. Bildgebung ist auch grundlegend für das frühzeitige Erkennen des individuellen Therapieerfolgs, um bei fehlendem Ansprechen die bisher eingeschlagene Behandlungskonzeption zu ändern. Auch die Entwicklung neuer Therapieverfahren basiert teilweise auf bildgebungsbasierten Biomarkern, die sowohl zum Einschluss vom Patienten in klinische Studien als auch als Endpunkt für den Therapieerfolg herangezogen werden.

Die Bildgebungsverfahren zur Ganzkörperdiagnostik (CT, MRT, PET) haben sich in den letzten 50 Jahren aufgrund der rasanten Fortschritte der Medizintechnik sowie der Computer- und Informationstechnologie erstaunlich weiterentwickelt. Mittlerweile können innerhalb kürzester Zeit nichtinvasiv genaue dreidimensionale Einblicke in anatomische und funktionelle Veränderungen des Körpers bildlich in hoher räumlicher Auflösung dargestellt und zugeordnet werden. Der Einsatz spezieller Radiopharmaka ermöglicht es, insbesondere auch tumorbiologische Eigenschaften, wie Stoffwechselaktivität und Rezeptordichte, zu visualisieren. „Hybridverfahren“ wie PET-CT und PET-MRT, mit denen sich die Vorteile von morphologischer und funktionell-biologischer Bildgebung kombinieren lassen, liefern damit in einem Untersuchungsgang individuell für jeden Patienten eine Vielfalt von therapierelevanten Informationen. Leistungsstarke Computer und Methoden der künstlichen Intelligenz ermöglichen es zudem, Algorithmen zu entwickeln, mit denen sich die immer umfangreicher werdenden, kom- plexen Datenmengen automatisiert verarbeiten lassen, und damit den Befundungsprozess zu unterstützen und zu beschleunigen. Die verschiedenen Beiträge des vorliegenden Schwerpunkthefts widmen sich diesen interessanten Entwicklungen einer erweiterten digitalen Bildgebung und molekularen Diagnostik in der Onkologie. Der Artikel von C. Dreher und S. Bickelhaupt zur Früherkennung und zum Screening zeigt insbesondere die Bedeutung der Bildgebung für die sekundäre und tertiäre Prävention auf. P. Sandach et al. widmen sich den Möglichkeiten der molekularen Bildgebung, die erhebliche Fortschritte bei der Entwicklung spezieller Radiotracer bzw. molekularer Sonden zur Visualisierung tumorspezifischer molekularer Strukturen und Stoffwechselveränderungen gemacht hat. Werden diese molekularen Sonden zudem an therapeutische Radionuklide gekoppelt (sog. Theranostik), erweitert sich das Feld der Diagnostik auf das der Therapie. F. Schaab et al. geben einen Überblick über radiologischinterventionelle Therapieverfahren, die zunehmend als minimalinvasive Strategien v. a. in der Palliativtherapie von Tumoren an Bedeutung gewinnen. Auch in der chirurgischen Onkologie werden zunehmend bildgebende Verfahren eingesetzt. Die Möglichkeiten und Bedeutung der modernen intraoperativen Bildgebung zur Visualisierung der dem $\mathrm{Au}$ ge bei der Operation zunächst nicht unmittelbar zugängigen Zielbereiche (wie z.B. intraparenchymale Tumorgrenzen) 
oder Risikostrukturen (z. B. zu schützende Gefäße oder Nerven) werden im Beitrag von L. Maier-Hein et al. auch insbesondere unter dem Aspekt einer Navigation chirurgischer Instrumente herausgearbeitet. Eine besondere Herausforderung stellt die Kommunikation erheblich großer Bilddaten- und Bildinformationsmengen dar. Diese Thematik wird von T. Persigehl et al. abgehandelt, und dabei werden die derzeitigen Entwicklungen einer strukturierten Befundung und eines Therapiemonitorings erörtert. F. Stögbauer et al. geben einen Einblick in die modernen Methoden der „liquid biopsy“, bei der molekulare Marker in Körperflüssigkeiten gemessen werden, um damit nicht-, bzw. minimalinvasiv Informationen über das Vorhandensein oder den Rückfall einer Tumorerkrankung zu erhalten. Abgerundet wird dieses Themenheft durch einen Artikel von J. Kleesiek et al. zum Thema der künstlichen Intelligenz und des maschinellen Lernens in der Radiologie. Die jüngsten Fortschritte der Computertechnologie und Bioinformatik lassen enorme, bisher nicht für möglich gehaltene Fortschritte auf diesem Gebiet erhoffen.

Dieses spannende Themenheft gibt somit den Lesern interessante Einblicke in die modernen Entwicklungen der digitalen Bildgebung und molekularen Diagnostik und deren Bedeutung für die klinische Onkologie. Unser besonderer Dank gilt daher den ausgewiesenen Autor*innen für ihre hierzu verfassten, hervorragenden Beträge. Diese vermitteln einen authentischen Überblick zu einem neuen, vielversprechenden diagnostischen Aufbruch in der Onkologie, letztendlich mit dem Ziel, die Prognose von Tumorpatienten weiter $\mathrm{zu}$ verbessern.

Für die Schriftleiter

H.-P. Schlemmer

Für die Herausgeber

P. M. Schlag

\section{Korrespondenzadresse}

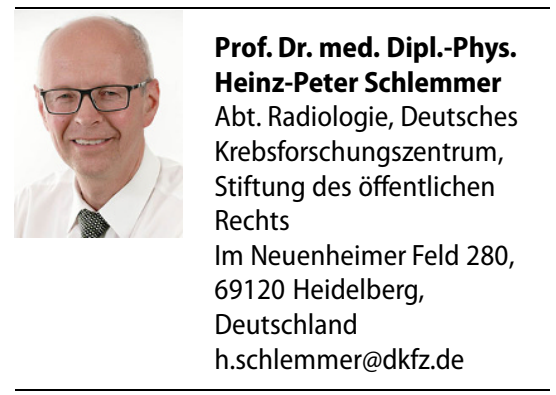

Interessenkonflikt. H.-P. Schlemmer und P.M. Schlag geben an, dass kein Interessenkonflikt besteht.
ONKO-Internetportal

Fokus USA - Neues von den Kongressen ASH und SABCS

Mit Interviews und Gesprächsrunden vom Jahrestreffen der American Society of Hematology (ASH) und dem San Antonio Breast Cancer Symposiums (SABCS) startet das ONKO-Internetportal in das neue Jahr. Mehr als 25.000 Hämatologen aus alle Welt kamen zum ASH-Kongress nach Orlando, um sich über aktuelle Forschungsergebnisse auszutauschen. Bewertungen ausgewählter Daten liefert das ONKO-Internetportal mit einer interdisziplinären Expertenrunde zum Multiplen Myelom und zwei Expertengesprächen zu Lymphomen, Leukämieerkrankungen und MDS. Unter www.krebsgesellschaft.de/ash_2019 analysieren Medizinerinnen und Mediziner die neuen Therapieoptionen und ordnen ihre Relevanz für die klinische Praxis ein. Themen sind u.a. neue vielversprechende Daten zur CAR-T-Zell-Therapie, Studien zur Charakterisierung und Reparatur von Mutationen sowie Kombinationstherapien.

Fast zeitgleich wurden auf dem weltweit größten Brustkrebskongress in San Antonio eine Vielzahl neuer Studienergebnisse vorgestellt. Das ONKO-Internetportal präsentiert Highlights und Kontroversen, u.a. zu den Themen Immuntherapie beim triple-negativen Mammakarzinom, neue Substanzen beim HER2-positiven Brustkrebs und zu den Ergebnissen der PEARLund HER2CLIMB-Studie. Die Expertenrunde Mammakarzinom diskutiert außerdem über die Behandlung mit Aromatase-Hemmern und der

Therapie mit CDK4/6-Inhibitoren. Alle Experten-Videos stehen ab sofort unter www.krebsgesellschaft.de/sabcs_2019 zum Download bereit.

Quelle: ONKO-Internetportal in Kooperation mit der Deutschen Krebsgesellschaft e.V. (DKG), www.krebsgesellschaft.de 